\title{
NITRATO EM ÁGUAS SUBTERRÂNEAS DO ESTADO DE SÃO PAULO
}

\author{
Marcia Regina STRADIOTO \\ Elias Hideo TERAMOTO \\ Hung Kiang CHANG
}

\begin{abstract}
RESUMO
A utilização de águas subterrâneas vem sendo incrementada na mesma proporção em que se observa a deterioração de sua qualidade, com a consequente perda de sua potabilidade. Dentre os agentes que contribuem para a perda de potabilidade dessas águas destaca-se o nitrato, que constitui o contaminante mais comum de águas subterrâneas, notadamente em regiões urbanas. Em razão da elevada solubilidade e estabilidade química do nitrato, a contaminação pode abranger grandes extensões. A principal fonte de contaminação por nitrato é o esgoto doméstico, particularmente onde o saneamento básico é incipiente ou a manutenção da rede de esgoto é insuficiente para prevenir o vazamento de volumes expressivos de esgoto em subsuperfície. Foram analisadas 840 amostras de águas subterrâneas, provenientes dos principais sistemas aquíferos (Cristalino, Tubarão, Guarani, Serra Geral, Bauru e Taubaté) distribuídos pelo território paulista, com vistas a apresentar um diagnóstico detalhado da contaminação por nitrato no estado. Os resultados foram comparados com o padrão de potabilidade estabelecido pela Portaria de Consolidação n ${ }^{\circ}$ 5/2017 do Ministério da Saúde e com o Valor de Prevenção estabelecido pela CETESB no documento "Qualidade das Águas Subterrâneas no Estado de São Paulo - 2016-2018". Das amostras analisadas, 70 (8,4\%) ultrapassam o valor máximo permitido pela Portaria e 159 (18,9\%) estão acima do valor de prevenção. Observa-se que o Sistema Aquífero Bauru é o mais impactado pela contaminação por nitrato, em razão de sua ampla extensão, sua natureza aflorante e grande número de poços que extraem água deste sistema. Na condição oposta, o Sistema Aquífero Guarani é o menos impactado, uma vez que a maior parte deste sistema está confinado, impedindo o ingresso de contaminação oriunda da superfície.
\end{abstract}

Palavras-chave: Contaminação por nitrato; Águas subterrâneas; Qualidade de águas subterrâneas; Sistema aquífero; Contaminação em áreas urbanas.

\section{ABSTRACT}

NITRATES IN GROUNDWATER OF THE STATE OF SÃO PAULO. As groundwater consumption increases, its quality and consequently its potability are proportionally affected. Nitrates stand out among the agents that contribute to the loss of groundwater potability, as they are the most common groundwater contaminants, notably in urban regions. Furthermore, because of their high solubility and chemical stability, nitrate contamination can extend over large areas. The main source of nitrate contamination is domestic sewage, particularly where basic sanitation is incipient, or maintenance of the sewerage system is inadequate to prevent significant sewage leakage to the subsurface. A total of 840 groundwater samples collected from the main aquifer systems distributed throughout the State of São Paulo (Cristalino, Tubarão, Guarani, Serra Geral, Bauru and Taubaté) were analyzed for this study, which aimed at a detailed diagnosis of the nitrate contamination in the State. The results were compared with the potability standards established by the Brazilian Ministry of Health in the Consolidation Ordinance $n^{\circ}$ 5/2017 and the Prevention Value established by CETESB in the document "Groundwater Quality in the State of São Paulo - 2016-2018". From 
the samples analyzed, $70(8.4 \%)$ exceeded the maximum value allowed by Ordinance $\mathrm{n}^{\mathrm{o}}$ 5, and 159 samples (18.9\%) exceeded the Prevention Value. The Bauru Aquifer System is the most affected by nitrate contamination, due to its size, its characteristics typical of free aquifers, and the large number of wells that extract water from this system. Conversely, the Guarani Aquifer System is the least impacted, because it is predominantly a confined aquifer system, preventing it from being affected by surficial contamination.

Keywords: Nitrate contamination; Groundwater; Groundwater quality; Aquifer system; Contamination in urban areas.

\section{INTRODUÇÃO}

A preocupação com a contaminação das águas subterrâneas vem crescendo, e um dos contaminantes antrópicos mais conhecidos é o nitrato, que pode atingir grandes áreas graças a sua alta mobilidade. O risco para a saúde humana ao consumir água com concentrações elevadas de nitrato-

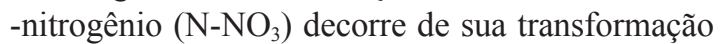
em nitrito no sistema digestivo. $\mathrm{O}$ nitrito oxida o ferro na hemoglobina dos glóbulos vermelhos e forma a metahemoglobina, que não possui a mesma capacidade de transporte de oxigênio da hemoglobina (WEITZBERG \& LUNDBERG 2013). Com isso, verifica-se a metahemoglobinemia (às vezes referida como «síndrome do bebê azul»), situação em que o sangue não tem a capacidade de transportar oxigênio suficiente para as células do corpo individuais, fazendo com que as veias e a pele se tornem azuis.

A contaminação das águas subterrâneas por nitrato foi associada ao emprego extensivo de fertilizantes em amplas áreas agrícolas em diversas regiões do mundo (GORMLY \& SPALDING 1979, BÖHLKE 2002, WANG et al. 2016, SPALDING et al. 2019). Por sua vez, o vazamento de esgoto em ambientes urbanos está igualmente entre as principais fontes de contaminação de águas subterrâneas por nitrato (MONTANHEIRO 2014, ZHANG et al. 2015, GRIMMEISEN et al. 2017, VYSTAVNA et al. 2017). No Brasil, são muitos os estudos descrevendo a contaminação de aquíferos por nitrato: CASTRO et al. (1992), MELO et al. (1998), GODOY et al. (2004), CABRAL et al. (2009), VARNIER et al. (2010), STRADIOTO et al. (2011), MOURA et al. (2013), MONTANHEIRO \& CHANG (2016), VICENTE et al. (2018), MARQUES et al. (2019), dentre outros.

Atualmente, a Companhia Ambiental do Estado de São Paulo (CETESB) possui uma rede de monitoramento com 313 poços tubulares e nascentes distribuídos por todo o estado. A partir desse mo- nitoramento, verificou-se em 2017 que as águas do Sistema Aquífero Bauru (SAB) apresentavam, em quatro amostras, nitrato acima do padrão de potabilidade e, em 41 amostras, concentração acima do valor de prevenção. Dos poços de monitoramento, 25 apresentaram concentrações entre $5 \mathrm{mg} / \mathrm{L}$ a 10 $\mathrm{mg} / \mathrm{L}$ de $\mathrm{N}-\mathrm{NO}_{3}$; dentre esses poços, 19 exploram água do Sistema Aquífero Bauru (SAB), três do Sistema Aquífero Guarani (SAG) e três do Sistema Aquífero Cristalino (SAC) (CETESB 2018).

Tendo em vista que a água subterrânea é a principal fonte de água para abastecimento público e privado em muitos munícipios do Estado de São Paulo, e diante da abundância de trabalhos descrevendo casos de contaminação por nitrato nessas águas, é de grande interesse que as concentrações deste contaminante sejam mais amplamente avaliadas no contexto dos principais sistemas aquíferos aí explorados. Assim, no presente trabalho é apresentado um diagnóstico detalhado e abrangente dos cenários de contaminação dos principais sistemas aquíferos do território paulista, a partir da análise química de águas de 840 poços.

\section{SISTEMAS AQUÍFEROS NO ESTADO DE SÃO PAULO}

No Estado de São Paulo, os principais sistemas aquíferos são: o Aquífero Cristalino, unidade do Domínio Cristalino; os sistemas aquíferos Tubarão, Guarani, Serra Geral e Bauru, do Domínio Vulcano-Sedimentar e Taubaté. Os sistemas aquíferos mencionados respondem pela maior parte da água empregada para abastecimento público e privado na área estudada.

O Sistema Aquífero Bauru (SAB) comporta-se como unidade hidrogeológica de extensão regional, contínua, livre e localmente confinada (DAEE 1974, 1976, 1979), estendendo-se por uma área de aproximadamente $96.880 \mathrm{~km}^{2}$ no Estado de São Paulo; é delimitado pelo rio Paraná a oeste e noroeste, pelo rio Grande ao norte, pelo rio 
Paranapanema ao Sul e pelas áreas de afloramento da Formação Serra Geral a leste (DAEE/IG/IPT/ CPRM 2005). Conforme proposta de PAULA E SILVA (2003) e PAULA E SILVA et al. (2006), o SAB divide-se em unidades hidroestratigráficas representadas pelos aquíferos Caiuá, Santo Anastácio, Birigui, Adamantina e Marília, e pelos aquitardos Pirapozinho e Araçatuba. As vazões registradas nas unidades do SAB são extremamente variáveis, em virtude da diversidade litofaciológica existente que coloca em contato lateral e vertical sedimentos com diferentes características de porosidade e permeabilidade (PAULA E SILVA 2003). Neste Sistema são definidas cinco zonas diferenciadas de vazão explorável, variando de 10 a $120 \mathrm{~m}^{3} / \mathrm{h}$, entretanto recomenda-se que as vazões praticadas não excedam $80 \mathrm{~m}^{3} / \mathrm{h}$ (DAEE/IG/IPT/CPRM 2005).

O Sistema Aquífero Serra Geral (SASG) é representado por um sistema fraturado formado pelos basaltos da Formação Serra Geral, do Eocretáceo, aflorando em uma área de cerca de $20.000 \mathrm{~km}^{2}$ na região central do Estado de São Paulo e ao longo dos principais rios que cortam o estado. Sua espessura varia de até $300 \mathrm{~m}$, na porção aflorante, a até $1.500 \mathrm{~m}$, em subsuperfície, aumentando de leste para oeste. Apresenta, em função de seu caráter descontínuo, grande variação nas vazões dos poços, que vão desde $1 \mathrm{~m}^{3} / \mathrm{h} / \mathrm{m}$ a $100 \mathrm{~m}^{3} / \mathrm{h} / \mathrm{m}$ (DAEE/IG/ IPT/CPRM 2005).

O Sistema Aquífero Guarani (SAG) ocupa a porção oeste do território paulista, em área de aproximadamente $174.000 \mathrm{~km}^{2}$, confinada pelos basaltos da Formação Serra Geral; estende-se em uma faixa aflorante de $16.000 \mathrm{~km}^{2}$, desde o município de Rifaina, ao norte, até o município de Fartura ao sul (DAEE/IG/IPT/CPRM 2005). No Estado de São Paulo o SAG é composto pelas formações Pirambóia (porção inferior) e Botucatu (porção superior). A Formação Pirambóia possui arenitos de granulação média a muito fina, localmente grossos e conglomeráticos, com estratificações cruzadas planar ou acanalada, de médio a grande porte, e estratificações plano-paralelas, predominantemente eólicas e subordinadamente fluviais; a Formação Botucatu, por sua vez, é composta por arenitos avermelhados de granulação média, em geral com grãos esféricos e bem selecionados, com estratificações cruzadas acanaladas de grande porte, depositados em um sistema eólico saturado (CAETANO-CHANG \& WU 1992, MILANI et al. 1994, CAETANO-CHANG \& WU 1996). As faixas de vazão recomendada são de $20 \mathrm{~m}^{3} / \mathrm{h}$ a $40 \mathrm{~m}^{3} / \mathrm{h}$ e $40 \mathrm{~m}^{3} / \mathrm{h}$ a $80 \mathrm{~m}^{3} / \mathrm{h}$, na porção livre, e de $80 \mathrm{~m}^{3} / \mathrm{h}$ a $120 \mathrm{~m}^{3} / \mathrm{h}, 120 \mathrm{~m}^{3} / \mathrm{h}$ a $250 \mathrm{~m}^{3} / \mathrm{h}$ e
$250 \mathrm{~m}^{3} / \mathrm{h}$ a $360 \mathrm{~m}^{3} / \mathrm{h}$, na porção confinada (DAEE/ IG/IPT/CPRM 2005).

O Sistema Aquífero Tubarão (SATU) é formado pelo Grupo Itararé e Formação Aquidauana, do Permo-Carbonífero, depositados em ambiente glacial continental e marinho, e pelo Grupo Guatá, do Permiano, constituído pelas formações Tatuí, Rio Bonito e Palermo, de ambiente marinho raso. O Grupo Itararé é formado por grande variedade de rochas, sendo reconhecidos diamictitos, ritmitos, siltitos, argilitos, folhelhos, conglomerados e arenitos acinzentados, cuja espessura total atinge aproximadamente $1.400 \mathrm{~m}$ (CAETANO-CHANG 1984). A Formação Aquidauana é composta por arenitos, conglomerados, siltitos e folhelhos, de ambiente glacial e flúvio-lacustre, chegando a $300 \mathrm{~m}$ de espessura. A Formação Tatuí, com maior expressão no Grupo Guatá, é formada principalmente por siltitos e em menor quantidade por arenitos, calcários, folhelhos e sílex. Sua faixa aflorante está localizada no centro-sudeste paulista, ocupando uma área de $20.700 \mathrm{~km}^{2}$. Devido à heterogeneidade deste sistema aquífero, as vazões variam entre 0 a $40 \mathrm{~m}^{3} / \mathrm{h}$, com predomínio de 0 a $10 \mathrm{~m}^{3} / \mathrm{h}$ (DAEE/IG/IPT/ CPRM 2005).

O Sistema Aquífero Taubaté (SAT), formado pelos sedimentos cenozoicos da Bacia de Taubaté, está localizado em uma depressão no embasamento pré-cambriano, com forma alongada este-nordeste, com $168 \mathrm{~km}$ de extensão por $20 \mathrm{~km}$ de largura, e área de aproximadamente $2.340 \mathrm{~km}^{2}$ (DAEE/IG/ IPT/CPRM 2005). O SAT ocorre de forma livre e pouco confinada, com raras áreas sob pressão (DAEE 1977). O Grupo Taubaté é formado por três formações: Resende, caracterizada por depósitos de arenitos, conglomerados, diamictitos e lamitos, de um sistema de leques aluviais associados a planícies aluviais de rios entrelaçados; Tremembé, constituída por argilitos, folhelhos, margas e calcários dolomíticos, lacustres; e São Paulo, composta por arenitos, argilitos, siltitos e arenitos conglomeráticos, de sistema fluvial meandrante (RICCOMINI 1989). A Formação Pindamonhangaba, sobreposta ao Grupo Taubaté, é formada por arenitos, conglomerados, argilitos e siltitos, de sistema fluvial meandrante, restrito ao centro da bacia (RICCOMINI 1989, MANCINI 1995).

O Aquífero Cristalino (SAC) é composto pelas rochas mais antigas do Estado de São Paulo, fraturado, de extensão regional de aproximadamente $53.400 \mathrm{~km}^{2}$, com área aflorante na porção leste paulista (IRITANI \& EZAKI 2009). Neste aquífero, os granitos e as rochas metamórficas possuem 
capacidade específica que varia de $0,01 \mathrm{~m}^{3} / \mathrm{h} / \mathrm{m}$ a $0,67 \mathrm{~m}^{3} / \mathrm{h} / \mathrm{m}$, enquanto as rochas carbonáticas, devido às suas altas porosidades, oriundas de dissolução, possuem capacidade específica superior, que varia de $0,12 \mathrm{~m}^{3} / \mathrm{h} / \mathrm{m}$ a $7 \mathrm{~m}^{3} / \mathrm{h} / \mathrm{m}$ (DAEE/IG/IPT/ CPRM 2005).

\section{3 ÁREA DE ESTUDO}

A área estudada compreende todo o território paulista (Figura 1), com $248.220 \mathrm{~km}^{2}$ e população estimada de 45.538 .936 pessoas em 2018 (IBGE 2019). As atividades econômicas principais são agricultura, pecuária, indústria, serviços, comércio e turismo. Possui grandes centros urbanos como São Paulo, Campinas, São Jose dos Campos, Sorocaba, Ribeirão Preto, entre outros.

Com importantes rios - Tietê, Grande, Piracicaba e Paranapanema -, o abastecimento público paulista divide-se em superficial e subterrâneo, sendo que em alguns municípios o abastecimento é exclusivamente subterrâneo. Estima-se que em $80 \%$ dos municípios paulistas a água subterrânea é a fonte principal ou única para abastecimento público. Na região oeste do estado, as águas do Sistema Aquífero Bauru (SAB), muitas vezes, tornam-se o único recurso hídrico para o abastecimento, enquanto na região leste, o uso das águas subterrâneas complementa o abastecimento público que na maioria dos casos, é realizado pela captação de águas superficiais (CETESB 2018).

\section{MATERIAIS E MÉTODOS}

\subsection{Amostras de águas subterrâneas}

Foram amostrados 840 poços de abastecimento entre os anos de 2003 a 2014; dentre esses, 592 (70,5\%) exploram água do SAB, $40(4,7 \%)$ do SASG, 65 (7,7\%) do SAG, 61 (7,3\%) do SATU, 63 (7,5\%) do SAC e 19 (2,3\%) do SAT, distribuídos em todo território paulista (Figura 1).

Previamente à coleta, as bombas existentes nos poços foram ligadas por alguns minutos para assegurar a renovação da água estagnada na tubulação e no poço. Para a amostragem, procurou-se um ponto de coleta próximo ao poço, porém anterior aos processos de fluoretação, cloração e/ou armazenamento da água. Todas as amostragens seguiram os padrões determinados paras coletas.

Imediatamente após a amostragem de água nos poços de abastecimento selecionados, procedeu-se à medição dos parâmetros físico-químicos, incluindo temperatura, condutividade elétrica e $\mathrm{pH}$ das amostras de água. Todas as amostras de água coletadas para análise de ânions e cátions foram fil-

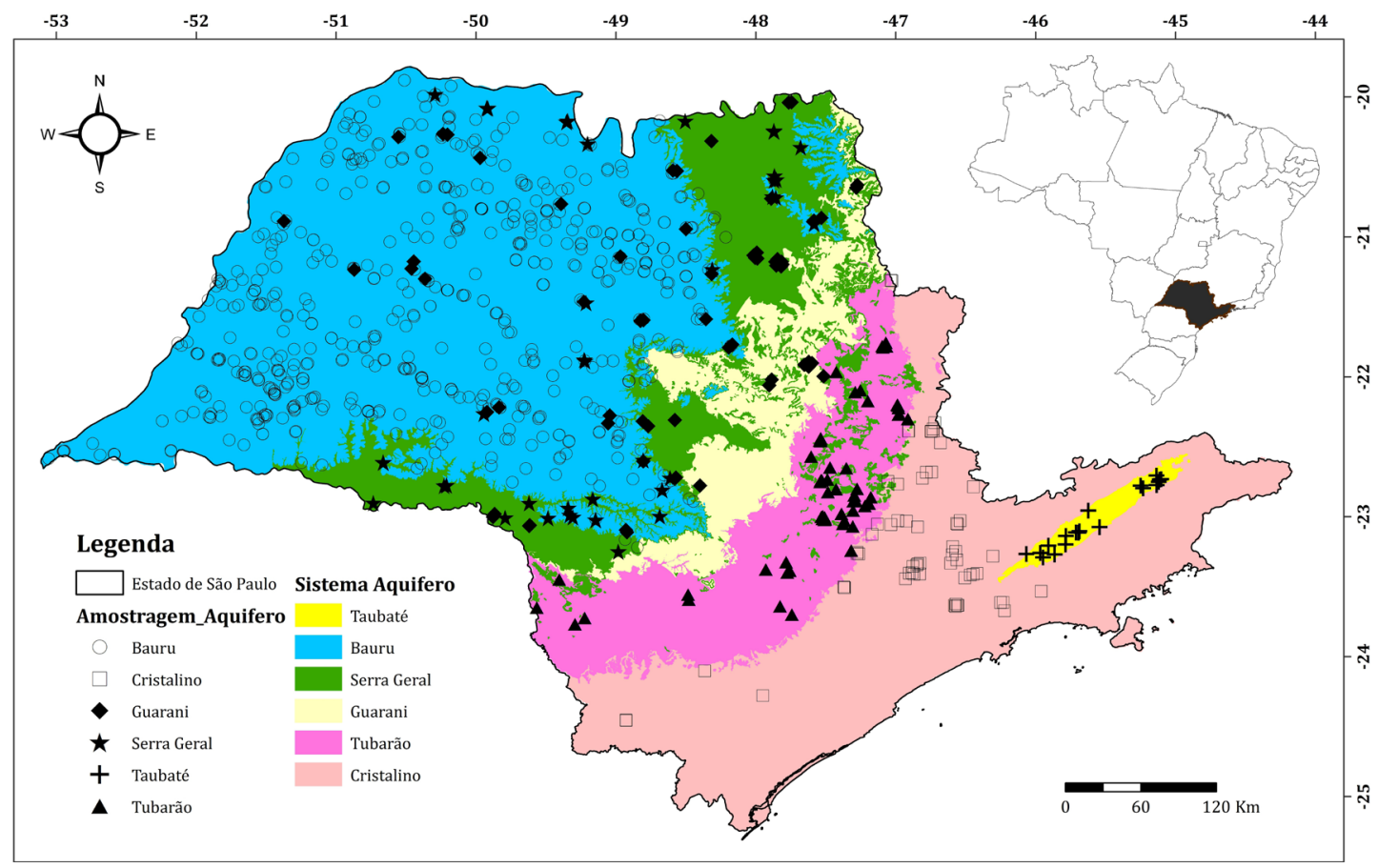

FIGURA 1 - Localização da área de estudo com a distribuição dos poços amostrados (Limites das unidades aquíferas aflorantes segundo DAEE 2013). 
tradas em um conjunto Millipore ${ }^{\mathrm{MR}}$, utilizando filtros de $0,45 \mu \mathrm{m}$; para análise de cátions, as amostras foram acidificadas com ácido nítrico concentrado (7,2 molar), com duas gotas para cada $50 \mathrm{~mL}$ de amostra. Após todos os procedimentos de coleta, os frascos foram fechados e conservados sob refrigeração até serem descarregados no laboratório.

Para determinação dos elementos $\mathrm{Ca}$ e $\mathrm{Mg}$ foi utilizado o método de espectrometria de emissão óptica, com fonte de plasma de argônio indutivo (ICP-OES). A alcalinidade $\left(\mathrm{HCO}_{3}{ }^{-} \mathrm{e} \mathrm{CO}_{3}{ }^{2-}\right)$ foi obtida por titulação, os ânions $\left(\mathrm{Cl}^{-}, \mathrm{NO}_{3}{ }^{-} \mathrm{e} \mathrm{SO}_{4}{ }^{2-}\right)$ e os cátions $\left(\mathrm{Na}^{+}\right.$e $\left.\mathrm{K}^{+}\right)$foram determinados por cromatografia de íons (IC), que seguiu o padrão estabelecido por Standard Methods for the Examination of Water and Wastewater (APHA/AWWA/WEF 2012). As análises químicas foram efetuadas no Laboratório de Hidroquímica do IGCE e no Centro de Estudos Ambientais (CEA), UNESP, campus de Rio Claro.

\subsection{Análise estatística}

Para uma avaliação quantitativa das concentrações de nitrato observadas no conjunto amostral avaliado, bem como o diagnóstico comparativo entre os diversos sistemas aquíferos estudados, foram realizadas análises estatísticas paramétricas, com a identificação dos parâmetros de tendência central (média, mediana e moda) e dispersão (desvio-padrão). Adicionalmente, foram construídos histogramas das concentrações de nitrato para avaliar a distribuição dos valores de concentrações nos diversos sistemas aquíferos estudados.

Para testar a premissa de que as concentrações de nitrato são positivamente correlacionáveis com as concentrações de cloreto e negativamente correlacionáveis com a profundidade dos poços amostrados, foram conduzidas análises de regressão linear para avaliar a existência de dependência estatística entre estes parâmetros.

\section{RESULTADOS E DISCUSSÃO}

O nitrato nas águas subterrâneas paulistas ocorre em diversas concentrações, variando de 0 a $41,6 \mathrm{mg} / \mathrm{L}$ de $\mathrm{N}^{-\mathrm{NO}_{3}}$ (Figura 2). Das 840 amostras, 70 apresentaram valor acima do máximo permitido para o padrão de potabilidade (Portaria de Consolidação $n^{\circ} 5 / 2017$ ) e 159 acima do valor de prevenção estabelecido pela CETESB (2018) (Figura 3), 112 apresentaram valores nulos ou abaixo do limite de detecção da técnica empregada $\left(0,04 \mathrm{mg} / \mathrm{L}\right.$ para $\mathrm{NO}_{3}$, admitindo valores nulos para este resultado na interpretação dos dados).

As amostras com concentração de nitrato acima do padrão de potabilidade $\left(10 \mathrm{mg} / \mathrm{L} \mathrm{N}-\mathrm{NO}_{3}\right)$

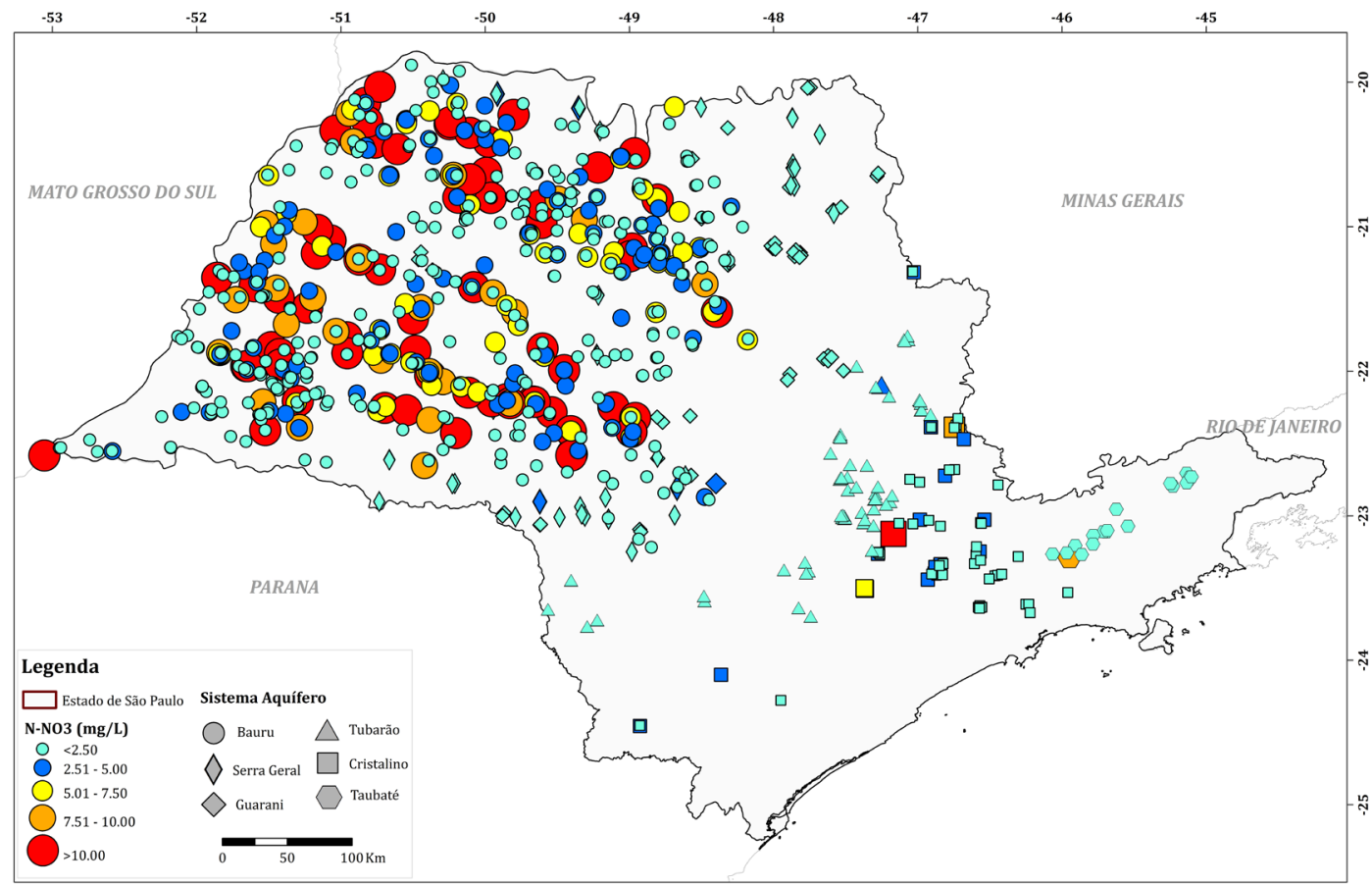

FIGURA 2 - Distribuição das concentrações de nitrato $\left(\mathrm{N}^{\left.-\mathrm{NO}_{3}\right)}\right.$ nas águas subterrâneas do Estado de São Paulo. 


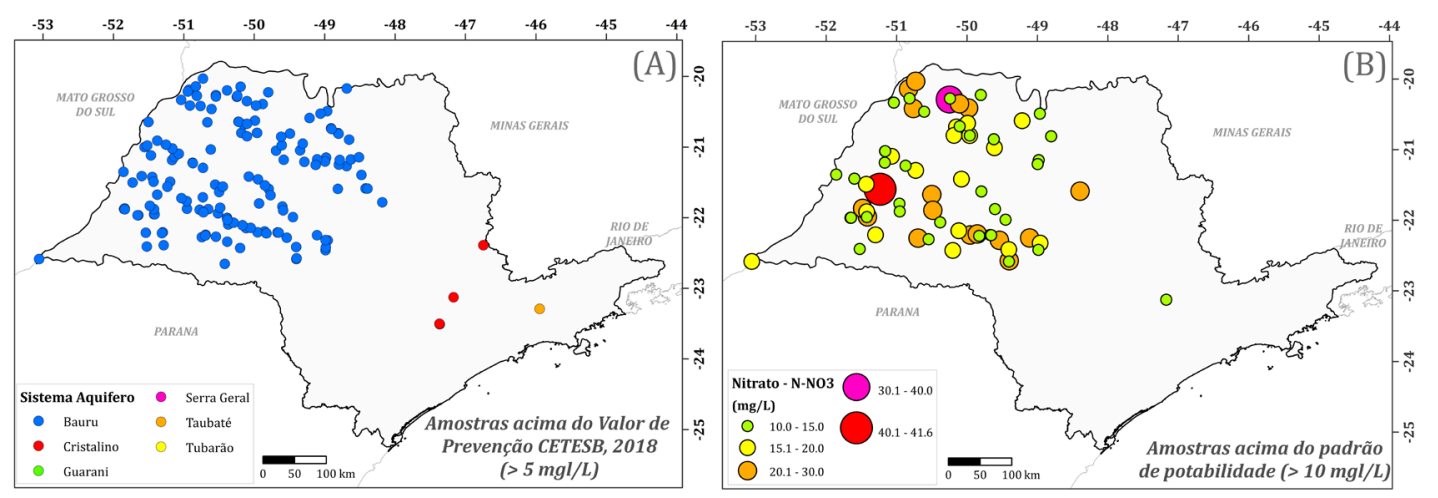

FIGURA 3 - Distribuição das concentrações de nitrato $\left(\mathrm{N}-\mathrm{NO}_{3}\right)$. (A) Amostras acima do valor de prevenção. (B) Amostras acima do valor de potabilidade.

foram predominantemente coletadas em poços que exploram o $\mathrm{SAB}$ e estão distribuídas por toda área deste sistema aquífero (Figura 3B). Dentre as amostras coletadas no SAB, $26 \%$ estão acima de 5 $\mathrm{mg} / \mathrm{L}$ (Valor de Prevenção) e a média é de 4,0 mg/L (Figura 4). Este sistema aquífero possui características livre a semiconfinado e uma grande área aflorante em território paulista, estando, assim, mais exposto à contaminação antrópica.

No SASG, as concentrações de $\mathrm{N}-\mathrm{NO}_{3}$ variam de 0 a 3,6 mg/L, com média de $0,8 \mathrm{mg} / \mathrm{L}$. As concentrações de $\mathrm{N}^{-\mathrm{NO}_{3}}$ no SAG variam de 0 a 4,8 $\mathrm{mg} / \mathrm{L}$, com média de $0,3 \mathrm{mg} / \mathrm{L}$; as maiores concentrações foram encontradas na porção aflorante e em poços mais rasos. No SATU, os valores de nitrato
$\left(\mathrm{N}_{-} \mathrm{NO}_{3}\right)$ variam de 0 a $3,1 \mathrm{mg} / \mathrm{L}$, com média de 0,3 $\mathrm{mg} / \mathrm{L}$. Nesses três sistemas aquíferos não foram encontradas amostras com concentrações acima de 5 $\mathrm{mg} / \mathrm{L}$ (Figura 4).

No SAC, as concentrações de nitrato $\left(\mathrm{N}^{-\mathrm{NO}_{3}}\right)$ variam de 0 a $10,1 \mathrm{mg} / \mathrm{L}$, com $8 \%$ das amostras acima de $5 \mathrm{mg} / \mathrm{L}$, uma amostra acima do padrão de potabilidade e média de $1,8 \mathrm{mg} / \mathrm{L}$. Por sua vez, o SAT apresenta concentrações de $\mathrm{N}_{-} \mathrm{NO}_{3}$ variando de 0 a $9,8 \mathrm{mg} / \mathrm{L}$, com $95 \%$ abaixo de $0,5 \mathrm{mg} / \mathrm{L}$, portanto abaixo do valor de prevenção, e média de 0,6 $\mathrm{mg} / \mathrm{L}$ (Figura 4). Cabe ressaltar que neste aquífero uma amostra apresentou valor de $9,8 \mathrm{mg} / \mathrm{L}$, aproximando do valor máximo permitido do padrão de potabilidade.
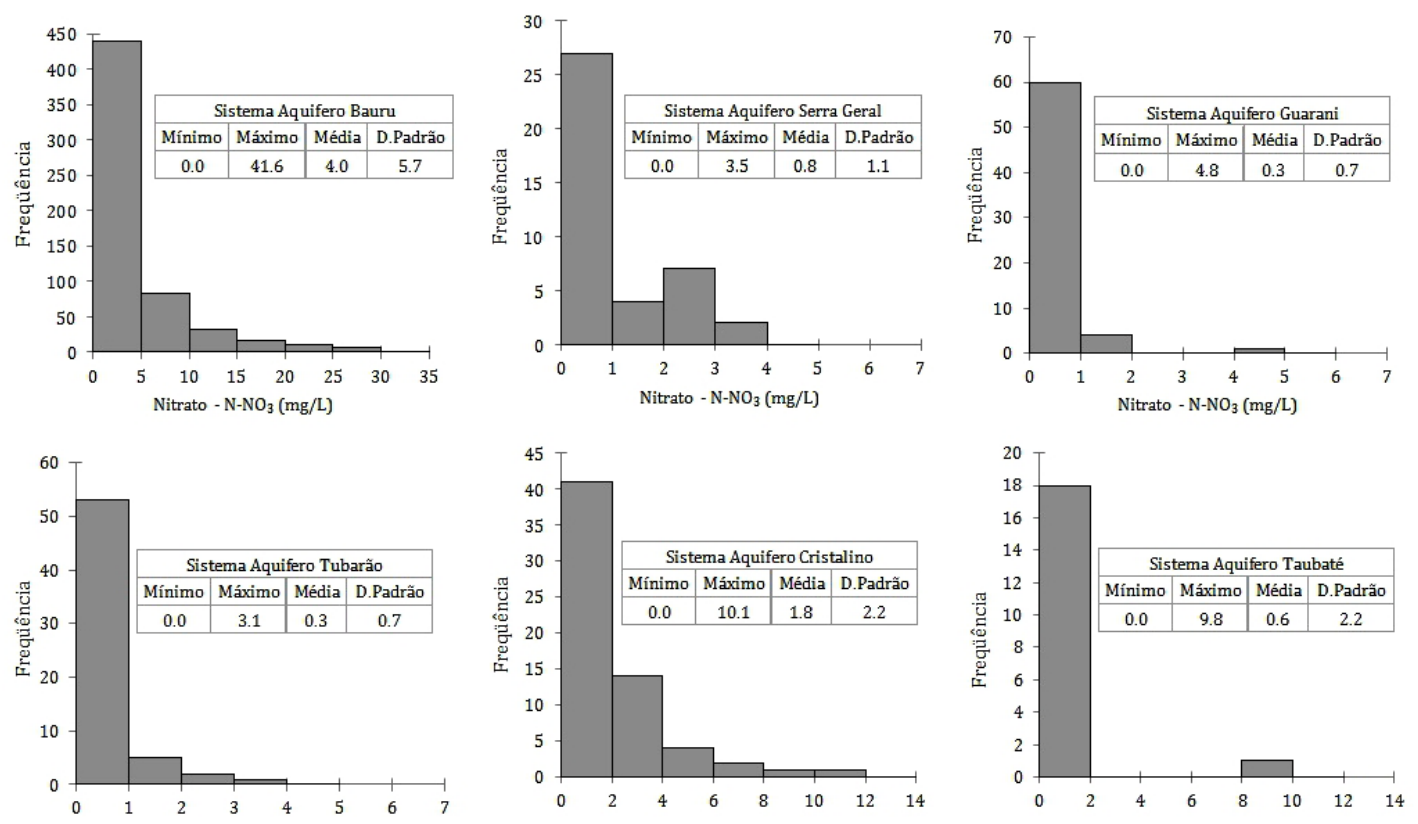

FIGURA 4 - Frequência das concentrações de Nitrato $\left(\mathrm{N}-\mathrm{NO}_{3}\right)$ de poços dos sistemas aquíferos no Estado de São Paulo. 
Os poços têm profundidades variáveis e muitas amostras foram coletadas na porção aflorante dos aquíferos, porém, a associação de nitrato com a profundidade dos poços amostrados não foi identificada (Figura 5A), uma vez que o coeficiente de correlação Pearson calculado para estes dois parâmetros foi de $-0,19$. Nota-se, especialmente no $\mathrm{SAB}$, que a relação nitrato com profundidade não é observada, sendo encontrados valores similares, com altas concentrações de nitrato, em poços rasos $\left(\mathrm{N}-\mathrm{NO}_{3}=21 \mathrm{mg} / \mathrm{L}\right.$; prof. $\left.=28 \mathrm{~m}\right)$ e profundos $(\mathrm{N}-$ $\mathrm{NO}_{3}=23 \mathrm{mg} / \mathrm{L}$; prof. $=234 \mathrm{~m}$ ). Contudo, observa-se que nos demais sistemas aquíferos, onde a maioria dos poços as profundidades são superiores a $150 \mathrm{~m}$, a contaminação por nitrato é menor.

As concentrações de cloreto alcançam valores de concentração bastante elevados, com máximo de $59,2 \mathrm{mg} / \mathrm{L}$. Não foi identificada correlação entre $\mathrm{Cl}^{-}$ e $\mathrm{NO}_{3}{ }^{-}$(Figura 5B); o coeficiente de correlação de Pearson calculado para esses ânions foi de 0,14 . É interessante notar que a concentração de $\mathrm{NO}_{3}{ }^{-}$possui baixa correlação com a profundidade dos poços e com a concentração de $\mathrm{Cl}^{-}$, contrariando as hipóteses originais. A expectativa inicial era que concentrações elevadas de $\mathrm{NO}_{3}{ }^{-}$fossem observadas em poços mais rasos, situados em aquíferos mais susceptíveis à contaminação. De maneira similar, era esperado que concentrações elevadas de $\mathrm{NO}_{3}{ }^{-}$fossem acompanhadas de concentrações igualmente elevadas do $\mathrm{Cl}^{-}$, uma vez que este íon, proveniente do sal de cozinha, bem como água sanitária, é observado em elevadas concentrações em efluentes domésticos. Tal correlação talvez possa ser melhor observada em escala local, como nos estudos realizados em Monte Azul Paulista (MONTANHEIRO \& CHANG 2016), Americana (MONTANHEIRO

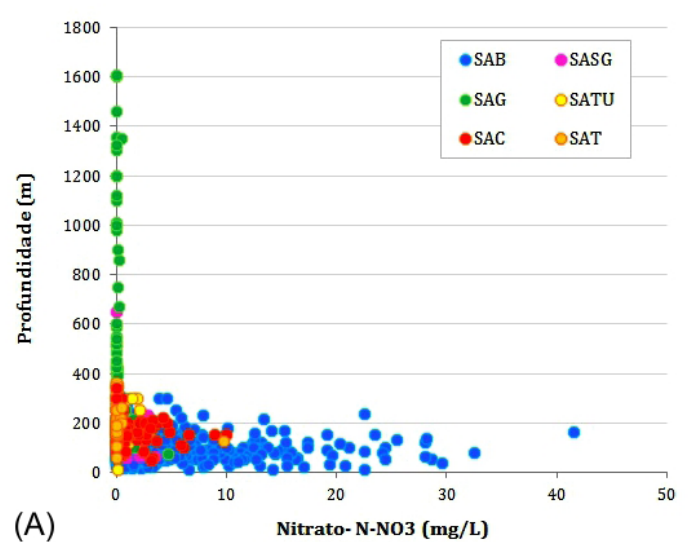

et al. 2014), Presidente Prudente (GODOY et al. 2004), Urânia (GAGNON 2003), entre outras áreas.

Os resultados indicam que a concentração do nitrato independe da profundidade, e que poços com profundidades superiores a $150 \mathrm{~m}$ podem apresentar concentrações de $\mathrm{NO}_{3}{ }^{-}$acima do limite de potabilidade. A baixa correlação entre $\mathrm{Cl}^{-} \mathrm{e}$ $\mathrm{NO}_{3}{ }^{-}$é decorrente da variabilidade da composição do efluente doméstico, bem como de concentrações naturalmente elevadas de $\mathrm{Cl}^{-}$em porções profundas do SAT, SAG e em aquíferos cristalinos situados em regiões costeiras.

Os resultados indicam que, como esperado $a$ priori, todos os sistemas aquíferos apresentam algum tipo de alteração em sua qualidade. Entretanto, nos sistemas aquíferos Tubarão (SATU), Guarani (SAG), Cristalino (SAC) e Taubaté (SAT) não foram observadas concentrações acima do limite de potabilidade, enquanto no Sistema Aquífero Cristalino (SAC) em apenas uma amostra foi encontrada concentração acima do limite estabelecido. No caso dos sistemas SATU e SAG, a condição de confinamento dificulta ou impossibilita que a contaminação os atinja em suas porções mais profundas. O SASG é representado por sucessivos derrames de basaltos, com alternância de horizontes permeáveis (porção superior e basal do derrame) e pouco permeáveis (interior do derrame), criando uma condição de confinamento que impede a contaminação dos horizontes permeáveis mais profundos. No caso do SAC, a ausência de concentrações elevadas de nitrato pode estar associada ao fato de que a água deste sistema aquífero é extraída de descontinuidades profundas; além disso, os poços de captação são revestidos na porção correspondente ao manto de alteração, fazendo com que a prová-

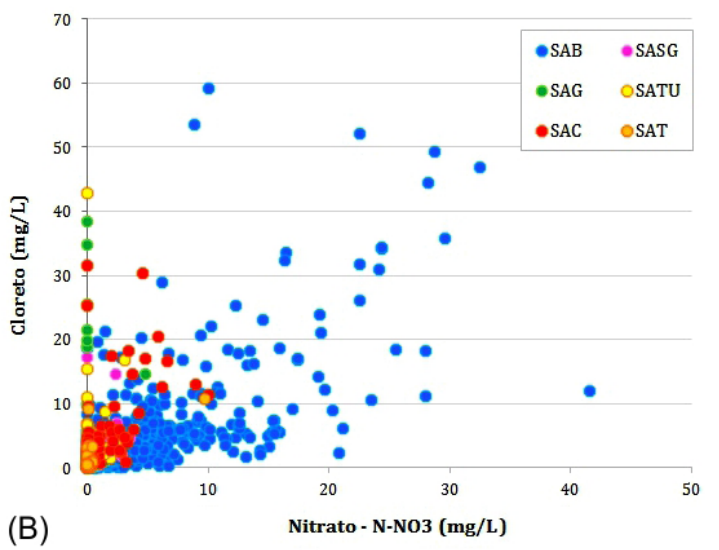

Nitrato - N-NO3 (mg/L)

FIGURA 5 - (A) Nitrato $\left(\mathrm{N}-\mathrm{NO}_{3}\right)$ versus profundidade. (B) Nitrato $\left(\mathrm{N}-\mathrm{NO}_{3}\right)$ versus Cloreto. 
vel água contaminada fique restrita às porções rasas e não seja extraída. Fato semelhante ocorre com o SAT, os poços amostrados geralmente estão em profundidades superiores a $150 \mathrm{~m}$, sendo menor a probabilidade da contaminação destas águas.

No caso do SAB, $11,48 \%$ das amostras possuem concentração de nitrato acima do valor de potabilidade (Tabela 1). Diversas amostras apresentaram concentrações duas a quatro vezes acima do valor de potabilidade, indicando casos bastante severos de contaminação. É relevante notar que a maior parte das concentrações elevadas de nitrato neste sistema é registrada no Aquífero Adamantina e menos frequentemente nos demais aquíferos que compõem o $\mathrm{SAB}$, as explicações seriam a grande área aflorante ocupada por este aquífero, maior quantidade de poços perfurados na região e sua elevada vulnerabilidade à contaminação, decorrente de sua elevada permeabilidade. Cabe destacar que os perfis construtivos da maior parte dos poços instalados no SAB indicam a existência de seção filtrante na porção superior da zona saturada, permitindo a extração de água em intervalos fortemente impactados pela contaminação por nitrato.

TABELA 1 - Número de amostras acima do limite de potabilidade para $\mathrm{N}-\mathrm{NO}_{3}$ para os diversos sistemas aquíferos estudados no Estado de São Paulo.

\begin{tabular}{ccc}
\hline $\begin{array}{c}\text { Sistema } \\
\text { Aquifero }\end{array}$ & $\begin{array}{c}\text { Porcentagem de amostras com } \\
\text { concentrações de } \mathrm{N}-\mathrm{NO}_{3} \text { acima } \\
\text { de } 10 \mathrm{mg} / \mathrm{L}(\%)\end{array}$ & $\begin{array}{c}\text { Número total } \\
\text { de amostras }\end{array}$ \\
\hline SAB & 11,48 & 592 \\
SASG & 0,00 & 40 \\
SAG & 0,00 & 65 \\
SATU & 0,00 & 61 \\
SAC & 0,02 & 63 \\
SAT & 0,00 & 19 \\
\hline
\end{tabular}

O impacto mais significativo do SAB decorre de sua ampla extensão em área, ocupando a maior parte da porção centro-oeste do Estado de São Paulo, e de ser um sistema aquífero raso, o que o torna mais susceptível à contaminação. Além disso, a contaminação do $\mathrm{SAB}$ pode ser associada à crescente urbanização verificada nas últimas décadas, além do extensivo uso agrícola, particularmente a cana-de-açúcar. A quase totalidade das águas do $\mathrm{SAB}$ foi amostrada em áreas urbanas, sendo plausível associar a contaminação com vazamento da rede de esgoto, em particular aquelas mais antigas. MARQUES et al. (2019) verificaram que a concen- tração de nitrato no $\mathrm{SAB}$, no munícipio de Urânia (SP), experimentou fortes incrementos nas últimas décadas, em razão da expansão da área urbana deste munícipio. Este cenário é compatível com aquele descrito por MONTANHEIRO \& CHANG (2016), no qual as concentrações de nitrato no SAB, no munícipio de Monte Azul Paulista (SP), são maiores nas porções urbanas mais antigas, decrescendo em direção às áreas urbanas mais novas. É interessante notar que a associação entre incremento da urbanização e elevação das concentrações de nitrato foi observada em outras regiões do mundo, como descrito por CABRAL et al. (2009), em Natal (RN), e por ZHANG et al. (2015), no sul da China. Assim, é possível assumir que o caso crítico de contaminação por nitrato observado no SAB foi gerado pelo crescimento da área urbana experimentada nas últimas décadas.

Um cenário de contaminação por nitrato bastante peculiar foi descrito por MONTANHEIRO \& CHANG (2016), que constataram que as águas contaminadas de aquíferos rasos migram para aquíferos mais profundos, quando subjacentes a aquitardos, a partir do espaço anular dos poços de abastecimento. Este cenário de contaminação cruzada impõe a necessidade de se avaliar rigorosamente os aspectos geológicos locais quando da instalação dos poços para fins de abastecimento, visando impedir a contaminação de aquíferos profundos. $\mathrm{O}$ isolamento da porção rasa e a instalação da seção filtrante apenas em porções profundas impediria o cenário de contaminação descrito por MONTANHEIRO \& CHANG (2016). Por esta razão, a definição do perfil construtivo do poço com base em modelos hidroestratigráficos locais deve representar um importante critério para concessão de outorga de exploração de água.

A eliminação do nitrato das águas subterrâneas após sua extração pode ser conduzida a partir de filtração por osmose reversa. Entretanto, tal solução é bastante dispendiosa do ponto de vista financeiro, o que limita a sua utilização. Por esta razão, a maior parte das soluções propostas se baseia no tratamento in situ do nitrato, a partir da sua redução para $\mathrm{N}_{2}$ em processo conhecido como desnitrificação. A maior parte dos estudos em campo, envolvendo a remedição do nitrato, se baseia na construção de barreiras reativas com materiais orgânicos ou ferro zero-valente para promover a redução heterotrófica ou autotrófica do nitrato (SCHIPPER et al. 2004, SU \& PULS 2007, ROBERTSON et al. 2008, SUHOGUSOFF et al. 2019). Outra alternativa que se mostrou promissora em testes conduzidos 
em campo foi a injeção de compostos orgânicos, tais como etanol (TARTAKOVSKY et al. 2002, ZHANG et al. 2006). O etanol injetado serve como fonte de carbono para o crescimento da atividade de bactérias desnitrificantes. A taxa de desnitrificação descrita por TARTAKOVSKY et al. (2002) foi de $1,2 \mathrm{mg}-\mathrm{N} /(\mathrm{L} / \mathrm{dia})$, enquanto ZHANG et al. (2006) identificaram um tempo de meia-vida do etanol em torno de 2,2 dias. Entretanto, tais soluções foram aplicadas para problemas em pequena escala e inexistem ações visando a remediação de aquíferos em escala regional. Por esta razão, tais opções não devem ser discutidas como ações imediatas para a mitigação da contaminação no SAB.

Diante do exposto, o diagnóstico de contaminação aqui identificado impõe a necessidade de ações estratégicas para identificação e eliminação de fontes de contaminação, com vistas a assegurar a potabilidade da água no futuro. Recomenda-se que ações concretas sejam abertamente discutidas dentro da política de gestão de recursos hídricos estadual. Dentre as ações imediatas que devem ser conduzidas estão a identificação e o conserto de rupturas em toda a rede de esgoto, associados a uma rigorosa manutenção preventiva. Além disso, devem ser considerados os aspectos hidroestratigráficos locais para definição dos aspectos construtivos dos poços, com vistas a evitar problemas de contaminação cruzada.

\section{CONCLUSÕES}

O amplo diagnóstico da contaminação por nitrato nos principais sistemas aquíferos paulistas revelou que apenas no Sistema Aquífero Bauru foram registrados casos bastante severos de contaminação, enquanto nos demais sistemas aquíferos nenhuma amostra apresentou concentrações de $\mathrm{N}-\mathrm{NO}_{3}$ acima do limite de potabilidade, com exceção de uma única amostra coletada no Sistema Aquífero Cristalino. A maior quantidade de pontos contaminados e as maiores concentrações de nitrato encontradas no Sistema Aquífero Bauru estão relacionadas à grande exploração deste sistema aquífero, à extensa área aflorante e à presença de grandes centros urbanos em sua área de ocorrência.

O cenário diagnosticado neste trabalho reforça a necessidade de eliminar os vazamentos da rede de esgoto e a desativação de fossas negras em áreas não atendidas pelo serviço de coleta de esgoto, bem como considerar aspectos hidroestratigráficos na instalação de poços, com o intuito de prevenir problemas de contaminação cruzada. $\mathrm{O}$ fato de a maior parte das amostras aqui utilizadas ser proveniente de poços de abastecimento público traz ainda maior relevância a este estudo, uma vez que envolve risco à saúde de populações inteiras que ingerem água proveniente desses poços.

\section{AGRADECIMENTOS}

Os autores agradecem ao Laboratório de Estudos de Bacias - LEBAC-DGA/UNESP -Rio Claro e à FUNDUNESP, pelo suporte técnico e financeiro para elaboração desse trabalho. Aos relatores da Revista do Instituto Geológico pelas sugestões que enriqueceram a conclusão deste artigo.

\section{REFERÊNCIAS BIBLIOGRÁFICAS}

APHA/AWWA/WEF - AMERICAN PUBLIC HEALTH ASSOCIATION, AMERICAN WATER WORKS ASSOCIATION and WATER ENVIRONMENT FEDERATION. 2012. Standard Methods for the Examination of Water and Wastewater, $1368 \mathrm{p}$.

BÖHLKE, J.K. 2002. Groundwater recharge and agricultural contamination. Hydrogeology Journal, 10 (1): 153-179. http://dx.doi. org/10.1007/s10040-001-0183-3

BRASIL. Ministério da Saúde. Portaria de Consolidação $\mathrm{N}^{\circ}$ 5. de 28 de setembro de 2017. Consolidação das normas sobre as ações e os serviços de saúde do Sistema Único de Saúde. Anexo XX. Diário Oficial da União: República Federativa do Brasil, Poder Executivo, Brasília, DF, n.190, 03.out.2017. Disponível em: Suplemento, p. 443-449. Disponível em: http://pesquisa.in.gov.br/imprensa/ jsp/visualiza/index.jsp?jornal=1040\&pagin $\mathrm{a}=1 \&$ data $=03 / 10 / 2017 \&$ total Arquivos $=716$. Acessado em nov. 2019.

CABRAL, N.M.T.; RIGHETTO, A.M.; QUEIROZ, M.A. 2009. Comportamento do nitrato em poços do aquífero Dunas/Barreiras nas explotações Dunas e Planalto, Natal, RN, Brasil. Revista Engenharia Sanitária e Ambiental, 14(3): 299-306. http://dx.doi. org/10.1590/S1413-41522009000300003

CAETANO-CHANG, M.R. 1984. Análise ambiental e estratigráfica do Subgrupo Itararé (PC) no sudoeste do estado de São Paulo. Instituto de Geociências, Universidade 
de São Paulo, São Paulo, Tese de Doutorado, $310 \mathrm{p}$.

CAETANO-CHANG, M.R.; WU, F.T. 1992. Bacia do Paraná: Formações Pirambóia e Botucatu. In: SBG, CONGRESSO BRASILEIRO DE GEOLOGIA, 37, São Paulo, Roteiro de excursão, 19 p.

CAETANO-CHANG, M.R.; WU, F.T. 1996. Piramboia and Botucatu formations (Tr-J Parana Basin, South America): sedimentation and stratigraphy. In: IUGS, INTERNATIONAL GEOLOGICAL CONGRESS, 30, Beijing, Proceedings, vol. 3, p. 1045.

CAGNON, F.A. 2003. Origem e hidroquímica do nitrato nas águas subterrâneas do Aquífero Adamantina em Urânica, SP. Instituto de Geociências - USP, São Paulo, Dissertação de Mestrado, $162 \mathrm{p}$.

CASTRO, S.C.S.; ARID, F.M.; DOS SANTOS, C.C.M.; SILVA, R.A.; CUNHA I.P. 1992. Contaminação por água subterrânea em São José do Rio Preto (SP): contaminação por nitratos. In: ABAS, CONGRESSO BRASILEIRO DE ÁGUAS SUBTERRÂNEAS, 7, Anais, Revista Águas Subterrâneas (Suplemento), 103-106.

CETESB - COMPANHIA AMBIENTAL DO ESTADO DE SÃO PAULO. 2018. Qualidade das águas subterrâneas no estado de São Paulo. CETESB, São Paulo, Boletim 2017 81 p. Disponível em: https://cetesb.sp.gov.br/ aguas-subterraneas/publicacoes-e-relatorios/

\section{DAEE - DEPARTAMENTO DE ÁGUA E ENER-} GIA ELÉTRICA. 1974. Estudo de águas subterrâneas: região administrativa 6 (Ribeirão Preto), São Paulo, Geopesquisadora - Tahal, $2 \mathrm{v}$.

DAEE - DEPARTAMENTO DE ÁGUA E ENERGIA ELÉTRICA. 1976. Estudo de águas subterrâneas, regiões administrativas 7, 8 e 9 (Bauru, São José do Rio Preto e Araçatuba). São Paulo, v. 1 e 2.

DAEE - DEPARTAMENTO DE ÁGUA E ENERGIA ELÉTRICA. 1977. Estudo de Águas Subterrâneas - Região Administrativa 3 (São José dos Campos). São Paulo, v. 1 e 3.

DAEE - DEPARTAMENTO DE ÁGUA E ENERGIA ELÉTRICA. 1979. Estudo de águas subterrâneas, regiões administrativas $10 \mathrm{e}$ 11(Presidente Prudente e Marília). São Paulo, v. 1 e 2.

DAEE - DEPARTAMENTO DE ÁGUA E ENERGIA ELÉTRICA. 2013. Águas Subterrâneas no Estado de São Paulo. Diretrizes de Utilização e Proteção. Departamento de Águas e Energia Elétrica, Instituto Geociências e Ciências Exatas, Laboratório de Estudo de Bacias, São Paulo, 44 p.

DAEE/IG/IPT/CPRM - DEPARTAMENTO DE ÁGUA E ENERGIA ELÉTRICA/ INSTITUTO GEOLÓGICO/INSTITUTO DE PESQUISA TECNOLÓGICAS/SERVIÇO GEOLÓGICO DO BRASIL. 2005. Mapa de águas subterrâneas do Estado de São Paulo. Escala: 1:1.000.000. G.A. Rocha (Coord. Geral), São Paulo, Conselho Estadual de Recursos Hídricos.

GODOY, M.C.T.F.; BOIN, M.N.; SANAIOTTI, D.C.; SILVA, J.B. 2004. Contaminação das águas subterrâneas por nitrato em Presidente Prudente - SP, Brasil. Revista do Instituto Adolfo Lutz, 63(2): 208-214.

GORMLY, J.R.; SPALDING, R.F. 1979. Sources and Concentrations of Nitrate-Nitrogen in Ground Water of the Central Platte Region, Nebraska. Groundwater, 17 (3): 291301. https://doi.org/10.1111/j.1745-6584.1979. tb03323.x

GRIMMEISEN, F.; LEHMANN, M.F.; LIESCH, T.; GOEPPERT, N.; KLINGER, J.; ZOPFI, J.; GOLDSCHEIDER, N. 2017. Isotopic constraints on water source mixing, network leakage and contamination in an urban groundwater system. Science of the Total Environment, 583: 202-213. https://doi. org/10.1016/j.scitotenv.2017.01.054

IBGE - INSTITUTO BRASILEIRO DE GEOGRAFIA E ESTATÍSTICA. População Estimada. Disponível em https://www.ibge.gov. br/estatisticas/sociais/populacao.html. Acessado em 16 jan. 2019.

IRITANI, M.A.; EZAKI, S. 2009. As Águas Subterrâneas do Estado de São Paulo. SMA/ IG, São Paulo, 2a ed., 104 p. (Cadernos de Educação Ambiental, 1).

MANCINI, F. 1995. Estratigrafia e Aspectos da Tectônica Deformadora da Formação Pin- 
damonhangaba, Bacia de Taubaté, SP. Instituto de Geociências, Universidade de São Paulo, São Paulo, Dissertação de Mestrado, 107 p. https://doi.org/10.11606/D.44.1995. tde-03092013-084815

MARQUES, C.H.G.; TERADA, R.; GALVÃO, P.; HIRATA, R. 2019. Evolução espacial e temporal da contaminação por nitrato no aquífero urbano de Urânia (SP). Revista Águas Subterrâneas, 33(3): 258-269. https://doi. org/10.14295/ras.v33i3.29524

MELO, J.G.; QUEIROZ, M.A.; HUNZIKER, J. 1998. Mecanismos e fontes de contaminação das águas subterrâneas de Natal/RN por nitrato. Revista Águas Subterrâneas, X Congresso Brasileiro de Águas Subterrâneas, 1-8.

MILANI, E.J.; FRANÇA, A.B.; SCHNEIDER, R.L. 1994. Bacia do Paraná. Boletim de Geociências da Petrobrás, 8(1): 69-82.

MONTANHEIRO, F. 2014. Contaminação por nitrato no aquífero Adamantina: o caso do município de Monte Azul Paulista/SP. Instituto de Geociências e Ciências Exatas, Universidade Estadual Paulista Júlio de Mesquita Filho, Rio Claro, Dissertação de Mestrado, 93 p. http://hdl.handle.net/11449/110572

MONTANHEIRO, F.; CHANG, H.K. 2016. Nitrato no Aquífero Adamantina: O caso do município de Monte Azul Paulista. Revista do Instituto Geológico, 37(2): 25-44. http://dx.doi. org/10.5935/0100-929X.20160007

MONTANHEIRO, F.; CHANG, H.K.; GASTMANS, D. 2014. Estudo hidroquímico do Sistema Aquífero Tubarão (SAT) no Município de Americana, SP. Revista do Instituto Geológico, 35(1): 31-45. https://doi. org/10.5935/0100-929X.20140003

MOURA, C.C.; GASTMANS, D.; CHANG, H. K.; MODESTO, R.P.; RODRIGUES, P.F.; RUBY, E.C. 2013. Avaliação das concentrações de nitrato no aquífero Bauru em áreas rurais. In: CONGRESSO INTERNACIONAL DE MEIO AMBIENTE SUBTERRÂNEO, 3, São Paulo, Anais, 4 p.

PAULA E SILVA, F. 2003. Geologia de Subsuperficie e Hidroestratigrafia do Grupo Bauru no Estado de São Paulo. Instituto de Geociências e Ciências Exatas, Universidade Estadual Paulista, Rio Claro, Tese de Doutorado,
166 p. https://repositorio.unesp.br/bitstream/ handle/11449/103040/silva_fp_dr_rcla.pdf

PAULA E SILVA, F.; CHANG, H.K.; CAETANO-CHANG, M.R.; STRADIOTO, M.R. 2006. Sucessão Sedimentar do Grupo Bauru na Região de Pirapozinho (SP). Revista Geociências, 25(1): 17-26

RICCOMINI, C. 1989. Rift Continental do Sudeste do Brasil. Instituto de Geociências, Universidade de São Paulo, São Paulo, Tese de Doutorado, 256 p. http://dx.doi. org/10.11606/T.44.1990.tde-18032013105507

ROBERTSON,W.D.;VOGAN, J.L.;LOMBARDO, P.S. 2008. Nitrate removal rates in a 15 -yearold permeable reactive barrier treating septic system nitrate. Groundwater Monitoring \& Remediation, 28(3): 65-72. https://doi. org/10.1111/j.1745-6592.2008.00205.x

SCHIPPER, L, BARKLE, G.F.: HADFIELD, J.C.; VOJVOLDIC-VUKOVIC, M.; BURGESS, C.P. 2004. Hydraulic constraints on the performance of a groundwater denitrification wall for nitrate removal from shallow groundwater. Journal of Contaminant Hydrology, 69(3-4): 263-279. https://doi. org/10.1016/S0169-7722(03)00157-8

SPALDING, R.F.; HIRSH, A.J.; EXNER, M.E.; LITTLE, N.A., KLOPPENBORG, K.L. 2019. Applicability of the dual isotopes $\delta^{15} \mathrm{~N}$ and $\delta^{18} \mathrm{O}$ to identify nitrate in groundwater beneath irrigated cropland. Journal of Contaminant Hydrology, 220: 128-135. https://doi. org/10.1016/j.jconhyd.2018.12.004

STRADIOTO, M.R.; CHANG, H.K.; PAULA E SILVA, F.; SILVA, S.R. 2011. Nitrato nas águas subterrâneas dos sistemas Aquíferos Bauru e Guarani, área urbana de Bauru - SP. In: ABAS, CONGRESSO INTERNACIONAL DE MEIO AMBIENTE SUBTERRÂNEO, 2, São Paulo, Anais (CD-ROM).

SU, C.; PULS, R.W. 2007. Removal of added nitrate in the single, binary, and ternary systems of cotton burr compost, zerovalent iron, and sediment: implications for groundwater nitrate remediation using permeable reactive barriers. Chemosphere, 67(8): 1653-1662. https://doi.org/10.1016/j. chemosphere.2006.09.059 
SUHOGUSOFF, A.V.; HIRATA, R.; ARAVENA, R.; ROBERTSON, W.D., FERRARI, L.C.K.M.; STIMSON， J.; BLOWES, D.W. 2019. Dynamics of nitrate degradation along an alternative latrine improved by a sawdust permeable reactive barrier (PRB) installed in an irregular settlement in the municipality of São Paulo (Brazil). Ecological Engineering, 138: 310-322. https://doi. org/10.1016/j.ecoleng.2019.08.001

TARTAKOVSKY, B.; MILLETTE, D.; DEL ISLE, S.; GUIOT, S.R. 2002. Ethanol-Stimulated Bioremediation of Nitrate-Contaminated Ground Water. Groundwater Monitoring \& Remediation, 22(1): 78-87. https://doi. org/10.1111/j.1745-6592.2002.tb00656.x

VARNIER, C.L.; IRITANI, M.A.; VIOTTI, M.; ODA, G.H.; FERREIRA, L.M.R. 2010. Nitrato nas águas subterrâneas do Sistema Aquífero Bauru, área urbana do município de Marília (SP). Revista do Instituto Geológico, 31(1-2): 1-21. http://dx.doi. org/10.5935/0100-929X.20100001

VICENTE，G.Z.; LIMA，C.G.R.; MARQUES, S.M. 2018. Variabilidade espacial e temporal do Nitrato e Cloreto no Sistema Aquífero Bauru, estado de São Paulo. Revista Águas Subterrâneas, 32(3): 295-306. https://doi. org/10.14295/ras.v32i3.29099

VYSTAVNA, Y. DIADIN, D.; YAKOVLEV, V.; HEJZLAR, J.; VADILLO, I.; HUNEAU, F.; LEHMANN, M. 2017. Nitrate contamination in a shallow urban aquifer in East Ukraine: evidence from hydrochemical, stable isotopes of nitrate and land use analysis. Environmental Earth Sciences, 76(13): 1-13. https://doi. org/10.1007/s12665-017-6796-1

WANG, L.; STUART, M.E.; LEWIS, M.A.; WARD, R.S.; SKIRVIN, D.; NADEN, P.S.; COLLINS, A.L.; ASCOTT, M.J. 2016. The changing trend in nitrate concentrations in major aquifers due to historical nitrate loading from agricultural land across England and Wales from 1925 to 2150. Science of the Total Environment, 542: 694-705. https://doi. org/10.1016/j.scitotenv.2015.10.127

WEITZBERG, E.; LUNDBERG, J.O. 2013. Novel aspects of dietary nitrate and human health. Annual Review of Nutrition, 33: 129-159. https://doi.org/10.1146/annurevnutr-071812-161159

ZHANG,Y.;KHAN,I.A.;CHEN,X.H.;SPALDING, R.F. 2006. Transport and degradation of ethanol in groundwater. Journal of Contaminant Hydrology, 82(3-4): 183-194. https://doi. org/10.1016/j.jconhyd.2005.09.007

ZHANG, Q.; SUN, J.; LIU, J.; HUANG, G.; LU, C. 2015. Driving mechanism and sources of groundwater nitrate contamination in the rapidly urbanized region of south China. Journal of Contaminant Hydrology, 182: 221-230. https://doi.org/10.1016/j. jconhyd.2015.09.009

\section{Endereço dos autores:}

Marcia Regina Stradioto - Laboratório de Estudos de Bacias - UNESP, Campus de Rio Claro, Av. 24-A, 1515, Bairro Bela Vista, CEP 13506-900, Rio Claro, SP, Brasil.E-mail: marcia.stradioto@unesp.br

Elias Hideo Teramoto - Laboratório de Estudos de Bacias - UNESP e Centro de Estudos Ambientais UNESP, Campus de Rio Claro, Av. 24-A, 1515, Bairro Bela Vista, CEP 13506-900, Rio Claro, SP, Brasil. E-mail: elias.hideo-teramoto@unesp.br

Hung Kiang Chang - Laboratório de Estudos de Bacias - UNESP, Centro de Estudos Ambientais - UNESP e Departamento de Geologia Aplicada, Instituto de Geociências e Ciências Exatas - UNESP, Campus de Rio Claro, Av. 24A, 1515, Bairro Bela Vista, CEP: 13506-900, Rio Claro, SP, Brasil. E-mail: chang.hungkiang@unesp.br

Artigo submetido em 21 de outubro de 2019, aceito em 19 de dezembro de 2019. 